\title{
Geographic Differences on Clinical Manifestations of COVID-19 Infection Between Overseas Chinese and Local Chinese Patients
}

\author{
Yanhua Liang',* \\ Shanglong Kou ${ }^{1,2, *}$ \\ Xiaohe $\mathrm{Li}^{1}$ \\ Zhiyi Ke' \\ Rongrong Zou' \\ Yanchao Pan (iD) ${ }^{1,3}$ \\ Jing Yuan'
}

'Diagnosis and Treatment of Infectious Diseases Research Laboratory, Shenzhen Third People's Hospital, Shenzhen, People's Republic of China; ${ }^{2}$ College of Chemistry and Chemical Engineering, South China University of Technology, Guangzhou, People's Republic of China; ${ }^{3}$ Institute of Chemical Biology, Shenzhen Bay Laboratory, Shenzhen, People's Republic of China

*These authors contributed equally to this work
Correspondence: Yanchao Pan; Jing Yuan Diagnosis and Treatment of Infectious Diseases Research Laboratory, Shenzhen Third People's Hospital, 29 Bulan Road, Buji District, Shenzhen, 5I8II2, People's

Republic of China

$\mathrm{Tel}+860755-61222333$

Fax +86 0755-61238928

Email panychao@126.com;

sz_yuanjing@163.com
Introduction: The novel coronavirus (COVID-19) has become a global pandemic with sharp rises in the number of confirmed cases and rapid spread across the world. Here, we looked at the effects of geographic differences on clinical manifestations of SARS-CoV-2 infected patients.

Methods: A total of 114 confirmed COVID-19 patients were included in this study. The epidemiological, demographic, clinical, as well as laboratory findings were extracted from the electronic medical records of these patients.

Results: We report the observation that patients from overseas residents diagnosed with COVID-19 were mildly symptomatic with cough and presented with lower inflammatory response and attenuated virus clearance rate, as well as correspondingly prolonged days of hospital stay than local Chinese patients. Moreover, the receiver-operating characteristic (ROC) analysis, performed to provide a measure of the difference between two groups, showed that serum albumin had the highest area under the curve value $(0.81, p<0.001)$.

Discussion: Our results suggested that blood albumin level acted as a predictive value in distinguishing clinical features between local and overseas Chinese. This work underscores the need to identify distinguishably prognostic factors of geographical dissimilarity in COVID-19 patients.

Keywords: geographic differences, clinical manifestations, inflammatory responses, COVID-19 patients

\section{Introduction}

Since 30 January 2020, the coronavirus disease COVID-19 has been announced as a global public health emergency with almost 100 million confirmed infected cases and rising up to millions of deaths. Travel-related SARS-CoV-2 transmission has been demonstrated to promote the global prevalence of COVID-19 and affect the epidemic trajectory in the community. ${ }^{1,2}$ Many countries shut down all their external borders and implemented massive travel restrictions in attempts to mitigate the spreading of the COVID-19 pandemic. ${ }^{2,3}$ Some studies revealed the regional and country-wide mortality differences in communities. ${ }^{4-6}$ Here we looked at the effect of geographic difference on clinical manifestations of SARS-CoV-2 infected patients by comparison of overseas Chinese and local Chinese.

\section{Materials and Methods}

Coronavirus (2019-nCoV) RNA Detection Kit (PCR Fluorescence probing) approved by China's National Medical Products Administration, was used for SARS-CoV-2 
virus testing. Conditions for the amplifications include reverse transcription at $50^{\circ} \mathrm{C}$ for $15 \mathrm{~min}$, pre-denaturation at $95^{\circ} \mathrm{C}$ for $15 \mathrm{~min}$, followed by 45 cycles of $94^{\circ} \mathrm{C}$ for 15 $\mathrm{s}$ and $55^{\circ} \mathrm{C}$ for $45 \mathrm{~s}$ for fluorescence detection. The receiver operating characteristic curve analysis was used to determine the optimal threshold cut-off value. A cycle threshold $(\mathrm{Ct})$ value $\leq 40$ was defined as a positive test. Demographic and clinical data of involved patients were derived from electronic medical records. Routine blood tests were performed by Sysmex XT-2000i automated hematology analyzer (Sysmex Corporation). Inflammatory biomarker assays were run on ARCHITECT i2000 SR analyzer (Abbott Diagnostics) according to the protocols recommended by the manufacturer.

This study was conducted in accordance with the Declaration of Helsinki. The study was approved by Shenzhen Third People's Hospital Ethics Committee and the written informed consent was waived by the Ethics Commission. Any data we collected and analysed in this retrospective study were derived from clinical raw records without any intervention or influence on clinical treatment. No additional collection of human samples or genetic resource materials was performed in our study. All methods were carried out in accordance with relevant guidelines and regulations. To fully protect the privacies and rights of patients, only clinical data observations were used for publication and personal information will not be disclosed to any third party without patient's consent.

The Receiver Operating Characteristic (ROC) curve analysis was carried out to explore and compare the performance of haematological parameters. The area under the curve was calculated to assess the capability of each parameter on discriminating the differences between different comparisons. Mean ( \pm standard deviation [SD]) and range were reported for normally distributed, continuous variables. One-way ANOVA was used to compare continuous variables among three groups, while the post hoc test was applied with Tukey's method. All statistical tests were 2-tailed, and a $p$ value less than 0.05 was considered statistically significant.

\section{Results}

Enrolled from February 6 to April 62020, a total of 114 patients with confirmed COVID-19 by RT-PCR swab test, as previously described, ${ }^{7}$ were transferred to Shenzhen Third People's Hospital for medical isolation and treatment. Of the total, 50 of them were incoming passengers, including 40 Chinese overseas residents and students, as well as 10 foreigners, found to be SARS-CoV-2-positive during the two-week quarantine in place for arrivals in China. They were predominately from Western Europe (n $=30,60 \%)$ and North America $(n=13,26 \%)$, followed by South East Asia ( $\mathrm{n}=5,10 \%)$, as well as Eastern Europe ( $\mathrm{n}$ $=2,4 \%$ ). Twelve of them had a contact history with suspected cases of symptomatic individuals. Another 64 patients were local Chinese residents and 45 of them had a recent travel history to Hubei Province. Additionally, $72 \%$ of the travelers presented initial signs and symptoms within 3 days and 17 of them presented with fever or cough once they arrived. The local patients, mostly with fever $(\mathrm{n}=37,57.81 \%)$, were admitted to hospital with a median of 3 days (IQR: 1.25-6.39) since symptom onset. Statistical significances were identified on frequencies of cough (ANOVA, $p<0.001$ ) and phlegm (ANOVA, $p=0.033$ ) among participants, with more than three-fold of overseas Chinese patients having moderate or severe cough in comparison with local Chinese patients (as shown in Tables $1,50.00 \%$ vs $15.63 \%, p<0.001$ for Tukey's post-hoc test).

In general, there was no statistically significant difference on the level of immune-inflammatory parameters between foreign and overseas Chinese travelers. However, overseas Chinese patients and local Chinese patients presented considerable differences on several hematological markers during hospitalization. In the initial 3 days, the average levels of C-reactive protein (CRP) and interleukin-6 (IL-6) in the local Chinese group were both approximately twice as high as those of overseas Chinese patients $(p=0.039$ and $p=0.017$, respectively, as shown in Figure $1 \mathrm{~A}$ and $\mathrm{B})$. The attenuated level of serum IL-6 remained significantly higher in local Chinese patients for over a week $(4.46 \pm 2.65$ vs $2.74 \pm 1.54 \mathrm{pg} / \mathrm{mL}, p=0.043$ for Tukey's post-hoc test). Furthermore, in concomitance with the declines of proinflammatory cytokines during the following weeks of their hospitalization, there was an obviously sharp increase in the platelet count and a gradual decline of serum albumin (ALB) in local Chinese patients (Figure 1C and D), while the range of platelet counts was relatively stable over time in the overseas Chinese and foreign traveler groups. Subsequently, local Chinese patients recovered after an averaged 15.6 \pm 2.8 days of hospital stay, while that was dramatically prolonged for approximately another 10 days in the groups of overseas Chinese and foreign travelers with an average of $26.4 \pm 8.8$ days $(p<0.001)$ and $26.9 \pm 7.0$ days $(p<0.001)$, 


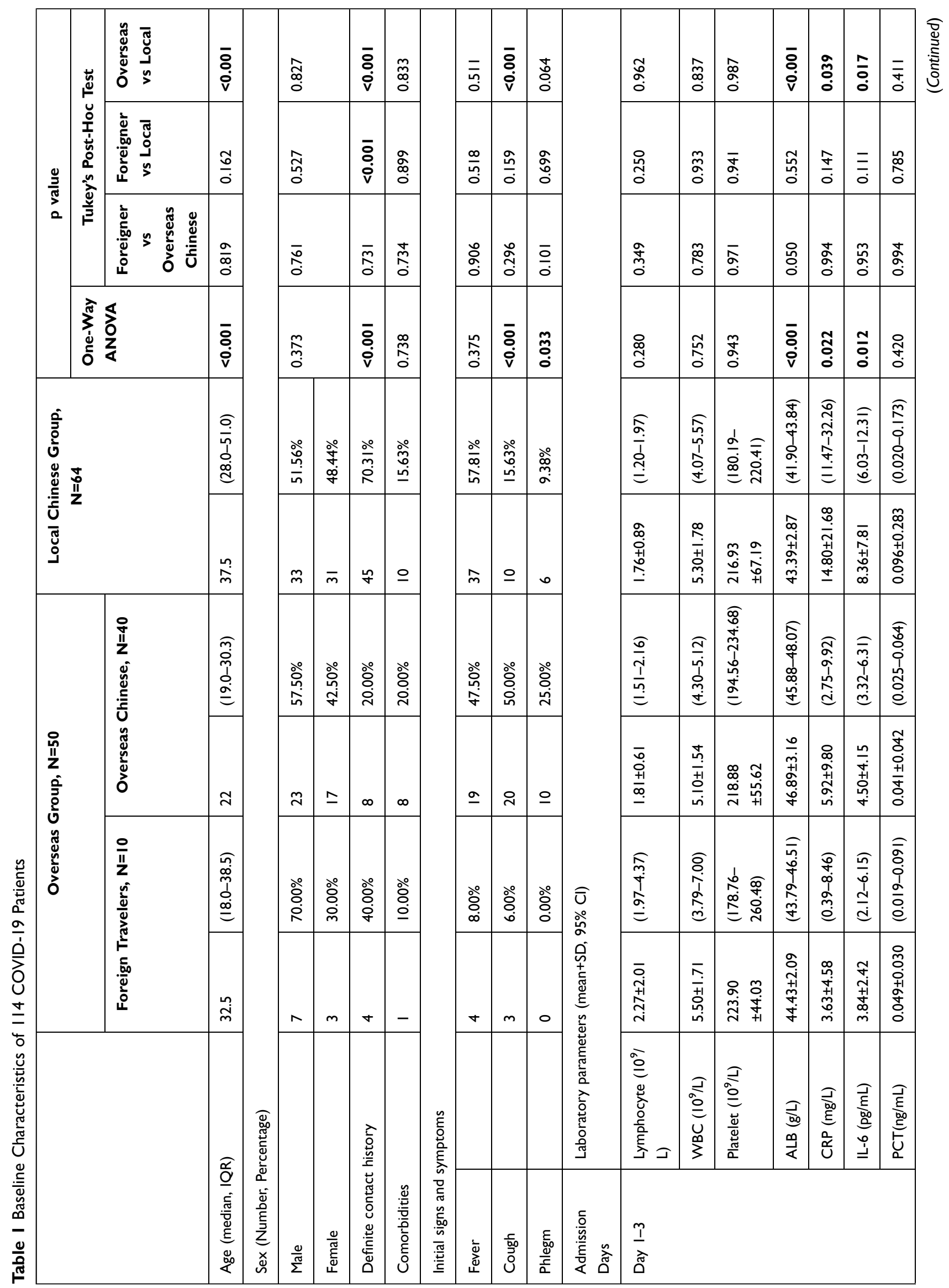




\begin{tabular}{|c|c|c|c|c|c|c|c|c|c|c|c|c|c|c|c|c|}
\hline \multirow{4}{*}{$\begin{array}{l}\frac{0}{\partial} \\
\frac{\partial}{2} \\
a\end{array}$} & \multirow{3}{*}{ 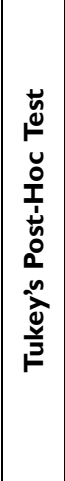 } & 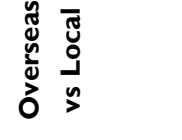 & స్తి & $\begin{array}{l}\text { h̊م } \\
\text { مै }\end{array}$ & $\frac{\bar{m}}{0}$ & $\begin{array}{l}\stackrel{2}{\Lambda} \\
\text { O }\end{array}$ & 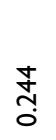 & $\begin{array}{l}\bar{\delta} \\
0 \\
0\end{array}$ & 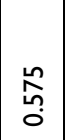 & $\begin{array}{l}\text { ò } \\
\text { o }\end{array}$ & $\begin{array}{c}\hat{0} \\
\text { o. } \\
0\end{array}$ & $\frac{n}{0}$ & $\begin{array}{l}\stackrel{0}{0} \\
0 \\
0\end{array}$ & 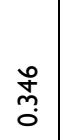 & $\begin{array}{l}\text { m } \\
\text { o } \\
0\end{array}$ & م̂م. \\
\hline & & 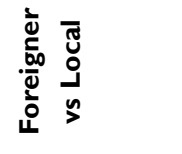 & సे & §o & $\frac{\sigma}{0}$ & $\stackrel{m}{n}$ & $\begin{array}{l}m \\
\stackrel{\sigma}{\sigma} \\
0\end{array}$ & 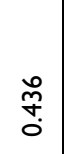 & $\begin{array}{l}\stackrel{L}{\alpha} \\
\sigma \\
o\end{array}$ & \begin{tabular}{l}
\multirow{2}{\sigma}{} \\
o. \\
0
\end{tabular} & $\begin{array}{l}\text { హ. } \\
\text { 。. }\end{array}$ & $\frac{\text { T }}{0}$ & $\frac{\grave{\Xi}}{0}$ & $\frac{\bar{\infty}}{\dot{0}}$ & $\underset{\stackrel{\circ}{\circ}}{\stackrel{0}{0}}$ & $\stackrel{\frac{m}{0}}{0}$ \\
\hline & & 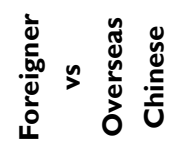 & 命 & \begin{tabular}{l}
\multirow{2}{*}{} \\
o
\end{tabular} & $\begin{array}{c}\stackrel{2}{\infty} \\
0 \\
0\end{array}$ & 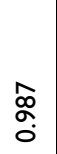 & $\begin{array}{l}\bar{\alpha} \\
\text { o. }\end{array}$ & $\begin{array}{l}\text { ôे } \\
\text { o. } \\
0\end{array}$ & $\begin{array}{l}\stackrel{0}{0} \\
0 \\
0\end{array}$ & $\begin{array}{l}0 \\
\alpha \\
\sigma\end{array}$ & 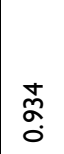 & $\begin{array}{l}\bar{\infty} \\
\stackrel{\alpha}{\circ}\end{array}$ & $\begin{array}{l}\hat{0} \\
\text { o. } \\
0\end{array}$ & $\begin{array}{l}\stackrel{\mathscr{0}}{0} \\
\stackrel{0}{0}\end{array}$ & 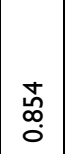 & $\stackrel{\stackrel{n}{\alpha}}{\tilde{m}}$ \\
\hline & \multicolumn{2}{|c|}{ 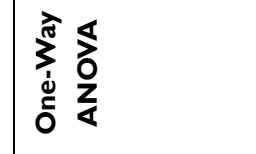 } & $\frac{a}{\infty}$ & 宮 & 苂 & $\frac{\Xi}{0}$ & ڤ્ণ & $\frac{\infty}{t}$ & $\begin{array}{l}\infty \\
0 \\
0 \\
0 \\
0\end{array}$ & $\begin{array}{l}\text { Jे } \\
\text { o. } \\
0\end{array}$ & $\stackrel{\substack{\infty \\
0 \\
0}}{0}$ & $\stackrel{\infty}{\circ}$ & $\begin{array}{l}\tilde{1} \\
0 \\
0\end{array}$ & $\frac{\Xi}{0}$ & ơ & 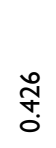 \\
\hline \multirow{2}{*}{\multicolumn{3}{|c|}{ 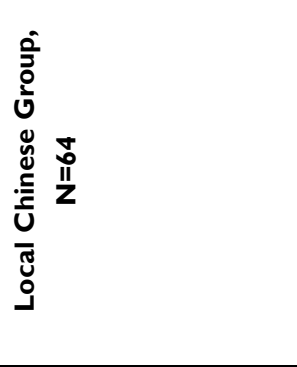 }} & $\begin{array}{l}\frac{\widehat{\omega}}{4} \\
\hat{T} \\
\stackrel{0}{=}\end{array}$ & 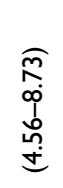 & 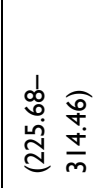 & 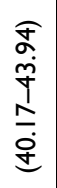 & 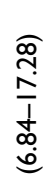 & $\begin{array}{l}\hat{\sigma} \\
\stackrel{\dot{m}}{b} \\
\stackrel{d}{d}\end{array}$ & 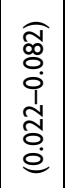 & $\begin{array}{l}\widehat{o} \\
\text { ì } \\
o \\
\stackrel{+}{=}\end{array}$ & 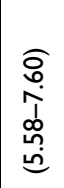 & 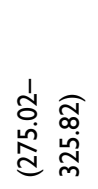 & 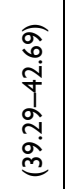 & $\begin{array}{l}\frac{\sigma}{0} \\
\frac{1}{a} \\
\frac{1}{d}\end{array}$ & 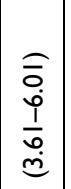 & 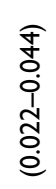 \\
\hline & & & 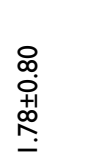 & 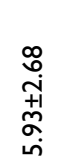 & 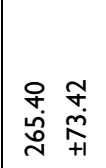 & $\begin{array}{c}\infty \\
\stackrel{\infty}{+} \\
\stackrel{+1}{+1} \\
\stackrel{0}{\dot{q}} \\
\dot{q}\end{array}$ & 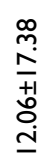 & $\begin{array}{l}\stackrel{m}{=} \\
\frac{\overline{+}}{a} \\
\stackrel{n}{n}\end{array}$ & 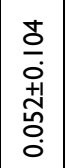 & $\begin{array}{l}a \\
0 \\
0 \\
0+1 \\
\vdots \\
\vdots \\
-1\end{array}$ & 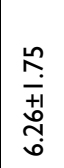 & 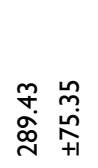 & 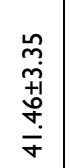 & $\begin{array}{l}\infty \\
0 \\
0 \\
+1 \\
\stackrel{1}{\sim} \\
\end{array}$ & 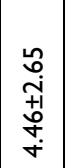 & 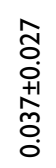 \\
\hline \multirow{4}{*}{ 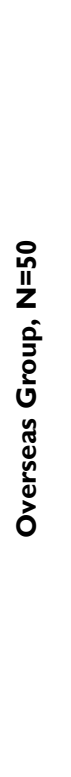 } & & \multirow{2}{*}{ 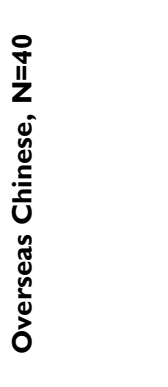 } & 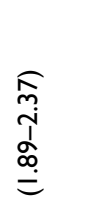 & $\begin{array}{l}\widehat{\sigma} \\
\underline{0} \\
\infty \\
o \\
\tilde{c}\end{array}$ & 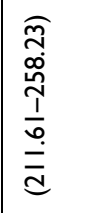 & 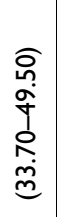 & $\begin{array}{l}\widehat{a} \\
\frac{0}{1} \\
\frac{1}{d} \\
\stackrel{+}{=}\end{array}$ & $\begin{array}{c}\frac{\widehat{o}}{a} \\
\dot{\omega} \\
\stackrel{+}{=}\end{array}$ & $\begin{array}{l}\widehat{\Phi} \\
\stackrel{0}{7} \\
\stackrel{1}{0} \\
\frac{1}{8} \\
\dot{0}\end{array}$ & $\begin{array}{l}\text { ָิ } \\
\hat{T} \\
\stackrel{N}{=}\end{array}$ & 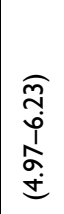 & 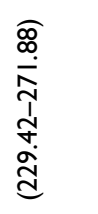 & 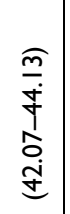 & 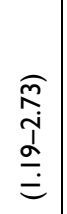 & $\begin{array}{c}\widehat{c} \\
\sim \\
0 \\
\infty \\
o \\
\stackrel{=}{=}\end{array}$ & 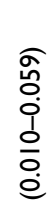 \\
\hline & & & $\begin{array}{l}o \\
0 \\
0 \\
+1 \\
0 \\
i \\
i\end{array}$ & 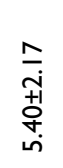 & $\begin{array}{l}\alpha \\
\sigma \\
\dot{j} \\
\tilde{\sigma} \\
\sigma \\
\sigma\end{array}$ & $\begin{array}{l}\hat{a} \\
m \\
+1 \\
o \\
\dot{y} \\
\dot{y}\end{array}$ & $\begin{array}{l}\frac{a}{j} \\
\frac{\mathrm{i}}{+1} \\
\frac{0}{0}\end{array}$ & 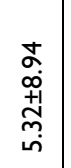 & $\begin{array}{l}\infty \\
m \\
0 \\
0+1 \\
+1 \\
o \\
0\end{array}$ & $\begin{array}{l}0 \\
\stackrel{0}{0} \\
+1 \\
0 \\
\text { o } \\
-1\end{array}$ & $\begin{array}{l}\text { L } \\
\text { ì } \\
\text { ơ } \\
\text { i }\end{array}$ & 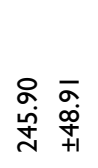 & 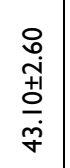 & $\begin{array}{l}a \\
\dot{+} \\
+1 \\
\stackrel{+}{j} \\
m\end{array}$ & 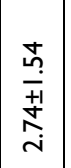 & 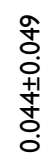 \\
\hline & & \multirow{2}{*}{ 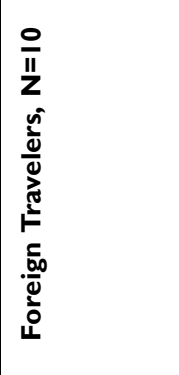 } & $\begin{array}{l}\text { ô } \\
\stackrel{+}{1} \\
\frac{\pi}{0}\end{array}$ & $\begin{array}{l}\widehat{0} \\
0 \\
0 \\
o \\
\stackrel{0}{0}\end{array}$ & 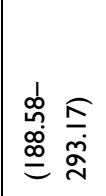 & $\begin{array}{l}\widehat{\bar{m}} \\
\dot{j} \\
\dot{j} \\
\alpha \\
\dot{j} \\
\dot{v}\end{array}$ & $\begin{array}{l}\widehat{\hat{र}} \\
\alpha \\
\infty \\
o \\
0\end{array}$ & $\begin{array}{l}\widehat{c} \\
\infty \\
0 \\
\infty \\
\infty \\
\stackrel{0}{0} \\
\stackrel{0}{0}\end{array}$ & 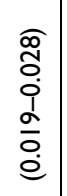 & 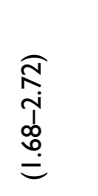 & $\begin{array}{l}\widehat{j} \\
o \\
\hat{j} \\
\hat{n} \\
\text { di }\end{array}$ & 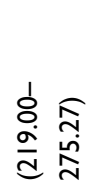 & 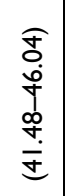 & 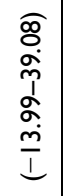 & $\begin{array}{c}\widehat{\widehat{N}} \\
\hat{\omega} \\
0 \\
\infty \\
\stackrel{0}{0}\end{array}$ & 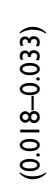 \\
\hline & & & $\begin{array}{l}\bar{m} \\
\stackrel{m}{+1} \\
\frac{m}{\dot{N}}\end{array}$ & 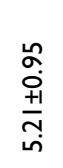 & 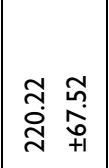 & 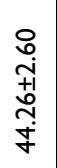 & $\begin{array}{l}\stackrel{0}{9} \\
\stackrel{0}{0} \\
\frac{0}{+1} \\
\stackrel{\rho}{m} \\
\stackrel{n}{n}\end{array}$ & 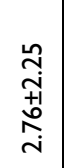 & 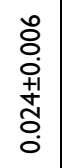 & 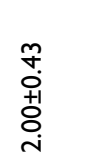 & 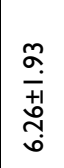 & 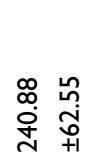 & 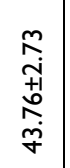 & 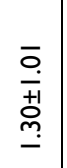 & 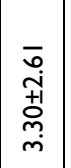 & 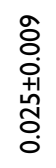 \\
\hline & & & 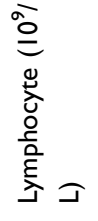 & 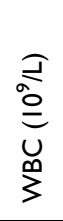 & 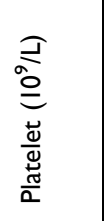 & 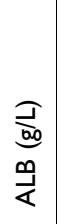 & 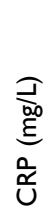 & 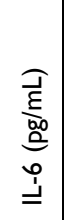 & 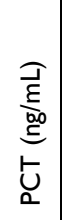 & 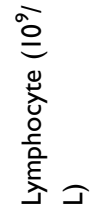 & 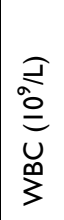 & 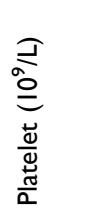 & 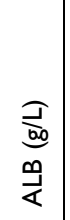 & 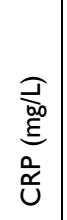 & 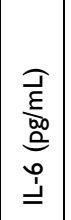 & 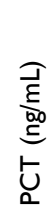 \\
\hline & & & $\begin{array}{l}\infty \\
+ \\
\text { 今̊ } \\
0\end{array}$ & & & & & & & $\begin{array}{l}\frac{n}{1} \\
\alpha \\
\widehat{A} \\
0\end{array}$ & & & & & & \\
\hline
\end{tabular}




\begin{tabular}{|c|c|c|c|c|c|c|c|}
\hline 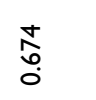 & $\begin{array}{l}\text { ठ } \\
\stackrel{0}{0}\end{array}$ & $\begin{array}{l}\text { ôे } \\
\text { f. } \\
0\end{array}$ & $\begin{array}{l}\bar{n} \\
\stackrel{0}{0}\end{array}$ & 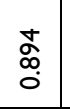 & $\overline{\overline{0}}$ & $\begin{array}{l}\infty \\
\vdots \\
0 \\
0\end{array}$ & $\begin{array}{l}\bar{o} \\
\dot{j} \\
\dot{v}\end{array}$ \\
\hline 亏ั & 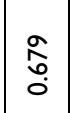 & $\begin{array}{l}\bar{R} \\
0\end{array}$ & $\stackrel{0}{\circ}$ & 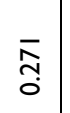 & $\frac{\varrho}{\bar{O}}$ & 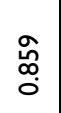 & $\begin{array}{l}\bar{o} \\
\dot{0} \\
\mathrm{v}\end{array}$ \\
\hline ב̀ & 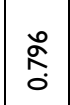 & $\begin{array}{l}\alpha \\
\text { o } \\
0 \\
0\end{array}$ & $\begin{array}{l}\underset{\sim}{0} \\
\stackrel{0}{0}\end{array}$ & $\frac{ \pm}{\frac{ \pm}{0}}$ & 孞 & 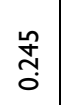 & $\begin{array}{l}\text { m} \\
\text { o. } \\
0\end{array}$ \\
\hline ồ & $\begin{array}{l}0 \\
\stackrel{2}{0} \\
0\end{array}$ & $\begin{array}{l}\bar{m} \\
\text { d. }\end{array}$ & $\begin{array}{l}\mathbf{1} \\
0 \\
0 \\
0\end{array}$ & $\frac{\varrho}{0}$ & \begin{tabular}{|l}
0 \\
$\vdots$ \\
0 \\
0
\end{tabular} & $\overline{\bar{o}}$ & $\begin{array}{l}\bar{\vdots} \\
\dot{v} \\
\dot{v}\end{array}$ \\
\hline 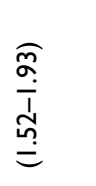 & 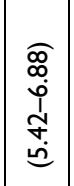 & 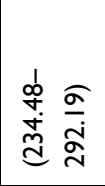 & 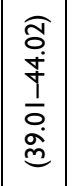 & 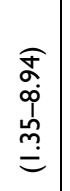 & 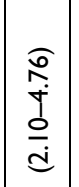 & $\begin{array}{l}\widehat{\Xi} \\
0 \\
0 \\
0 \\
0 \\
\dot{Q}\end{array}$ & 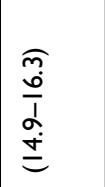 \\
\hline 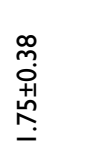 & $\frac{\stackrel{p}{m}}{\frac{+1}{\bar{t}}}$ & 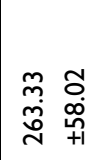 & $\mid$\begin{tabular}{c|}
0 \\
$h$ \\
+1 \\
$\frac{i}{\alpha}$ \\
$\dot{q}$ \\
\end{tabular} & 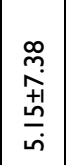 & 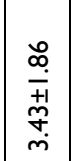 & 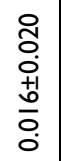 & 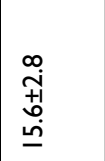 \\
\hline 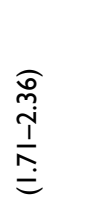 & 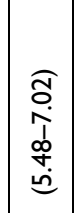 & 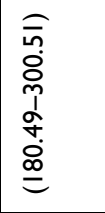 & 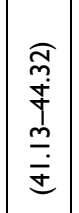 & 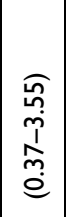 & 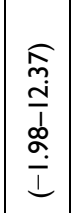 & 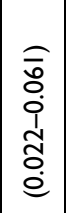 & 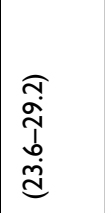 \\
\hline 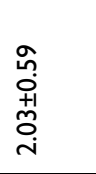 & 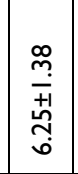 & 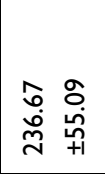 & 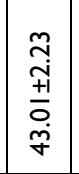 & 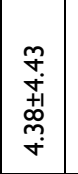 & 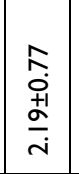 & 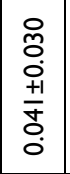 & \begin{tabular}{|l|}
$\infty$ \\
$\infty$ \\
0 \\
+1 \\
+1 \\
in \\
\end{tabular} \\
\hline 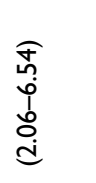 & 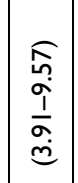 & 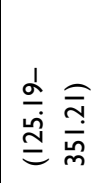 & 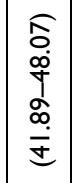 & 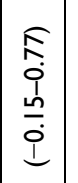 & 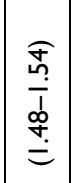 & 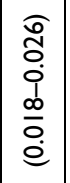 & $\begin{array}{l}\frac{\sigma}{\dot{m}} \\
\dot{\alpha} \\
\dot{\underline{d}}\end{array}$ \\
\hline 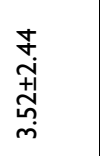 & 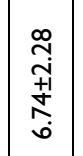 & 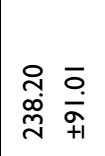 & 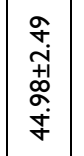 & \begin{tabular}{|c|}
$\hat{m}$ \\
$\hat{i}$ \\
$\frac{+1}{m}$ \\
$o$
\end{tabular} & 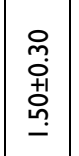 & 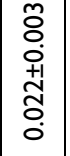 & $\begin{array}{l}0 \\
\stackrel{0}{0} \\
\text { +1 } \\
\text { di } \\
\text { in }\end{array}$ \\
\hline 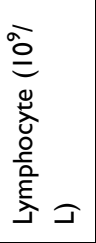 & 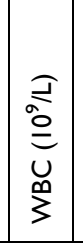 & 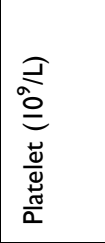 & 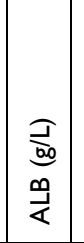 & 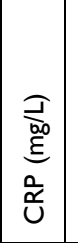 & 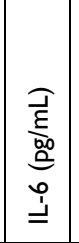 & 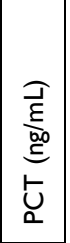 & 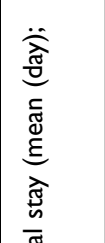 \\
\hline 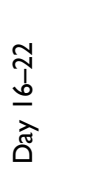 & & & & & & & 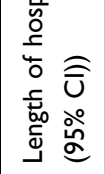 \\
\hline
\end{tabular}




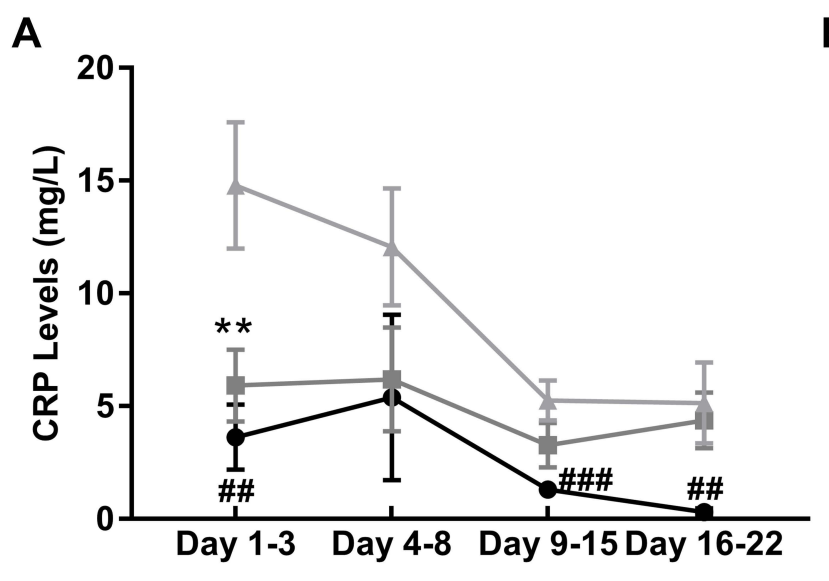

Hospital Stay

C

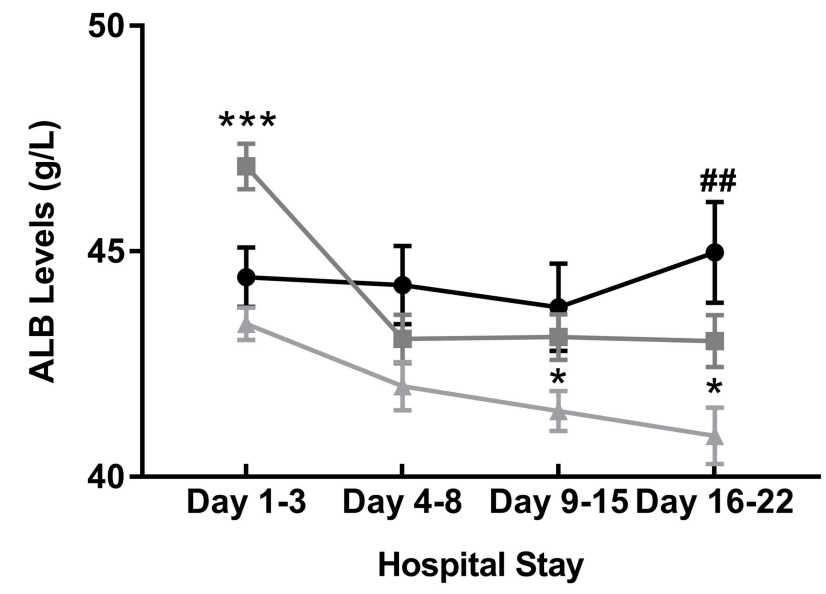

E

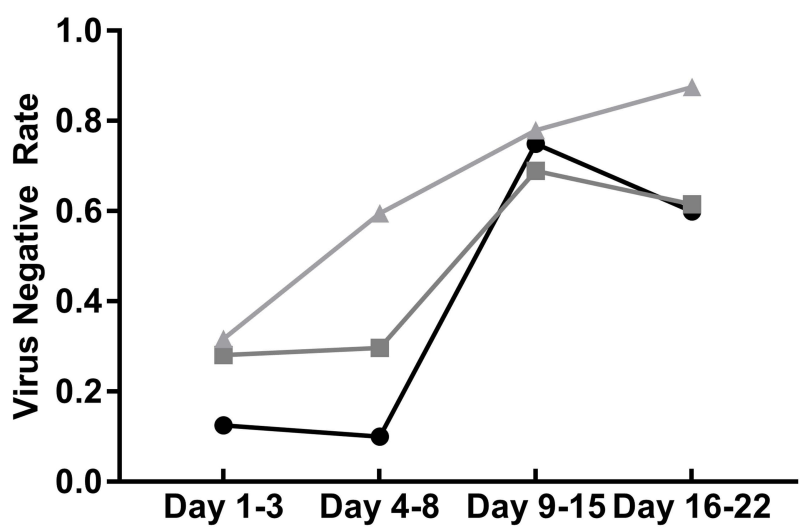

Hospital Stay
B

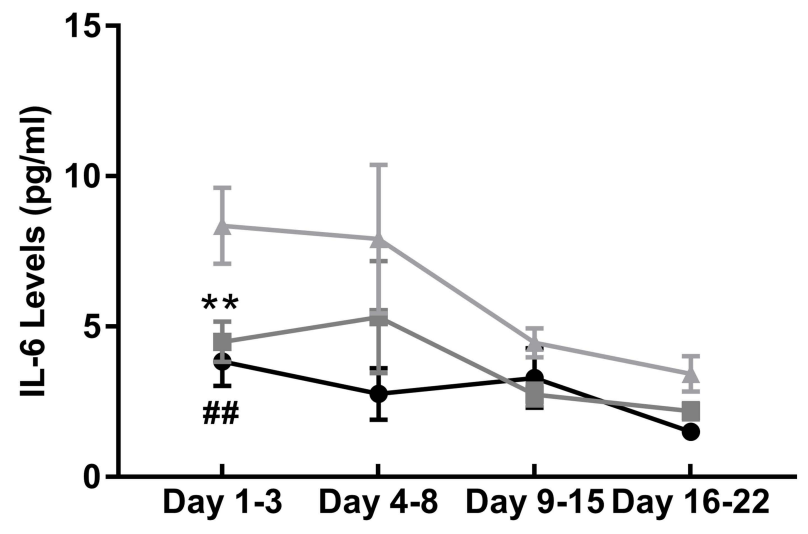

Hospital Stay

D

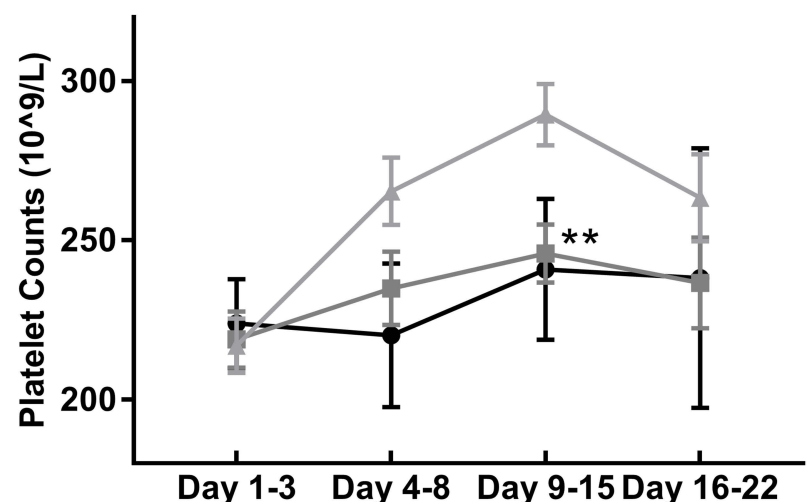

Hospital Stay

\section{$\rightarrow$ Foreign traveler \\ - Overseas Chinese \\ $\leftarrow$ Local Chinese}

Figure I Changes of laboratory parameters in the peri-hospitalization period. Comparisons on blood CRP (A) and IL-6 (B) indicated significantly higher inflammatory status in initial days of admission in local Chinese patients. Changes in ALB level $(\mathbf{C})$ were identified with distinguishing levels among three groups. The local patients also presented with an obvious increase of the platelet count (D) and virus clearance rate (E), which were attenuated in overseas Chinese and foreign travelers. The independent two samples $t$-test is used between two groups and statistical tests are 2-tailed. A p value less than 0.05 is considered statistically significant. The asterisks indicate significant differences between the oversea Chinese and local Chinese patients $\left({ }^{*} p<0.05,{ }^{* *} p<0.01\right.$, $\left.{ }^{* * *} p<0.00 \mathrm{I}\right)$; the hash symbol indicates a significant difference between the foreign traveler and local Chinese groups $\left({ }^{*} p<0.0 \mathrm{I}\right.$, $\left.{ }^{\prime \prime \prime} p<0.00 \mathrm{I}\right)$. Error bars: Mean \pm SD.

Abbreviations: ALB, Albumin; CRP, C-reactive protein; IL-6, Interleukin-6. 
respectively. Simultaneously, the group of foreign travelers exhibited with a significantly higher lymphocyte count of $3.52 \pm 2.4410^{9} / \mathrm{L}$ on average in comparisons with both overseas Chinese $\left(2.03 \pm 0.5910^{9} / \mathrm{L}, p=0.012\right)$ and local Chinese $\left(1.75 \pm 0.3810^{9} / \mathrm{L}, p=0.002\right)$. On the other hand, the local Chinese patients had a relatively lower serum ALB with an average $40.91 \pm 2.50 \mathrm{~g} / \mathrm{L}$ compared with the foreign travelers $(44.98 \pm 2.49 \mathrm{~g} / \mathrm{L}$, $p=0.006)$, as well as the overseas Chinese $(43.01 \pm 2.23$ $\mathrm{g} / \mathrm{L}, p=0.051)$.

Results of detection of SARS-CoV-2 (Figure 1E) by realtime reverse transcription polymerase chain reaction (RTqPCR) indicated that all the Chinese patients, including local and overseas ones, shared an equivalent capability of virus clearance initially. Subsequently, instead of the rapid rise in the proportion of patients with negative RT-PCR results in local Chinese group, overseas Chinese patients showed a suppression in the rate of virus clearance in the next week, followed by sharply rising up to a similar level of local Chinese patients contemporaneously. Remarkably, the virus negative rate in local Chinese patients was over $20 \%$ higher than that of the overseas group. These results corresponded with considerably prolonged days of hospital stay for overseas patients in comparison to local Chinese patients. In order to assess the prognostic value of initial inflammatory parameters, the Receiver Operator Characteristic (ROC) curve analysis of different cohorts (including incoming foreigners, overseas Chinese and local Chinese) were performed, showing that the area under the curve (AUC) for ALB level was 0.81 (95\% CI: $0.72-0.90, p<0.001)$ in comparison between the total overseas patients and local Chinese (Figure 2). Furthermore, the AUC derived from the comparison between overseas and local Chinese was the highest with a value of 0.83 (95\% CI: $0.74-0.92$, $p<0.001$ ), whereas that between incoming foreign patients and the total Chinese patients was 0.49 (95\% CI: 0.35-0.64,

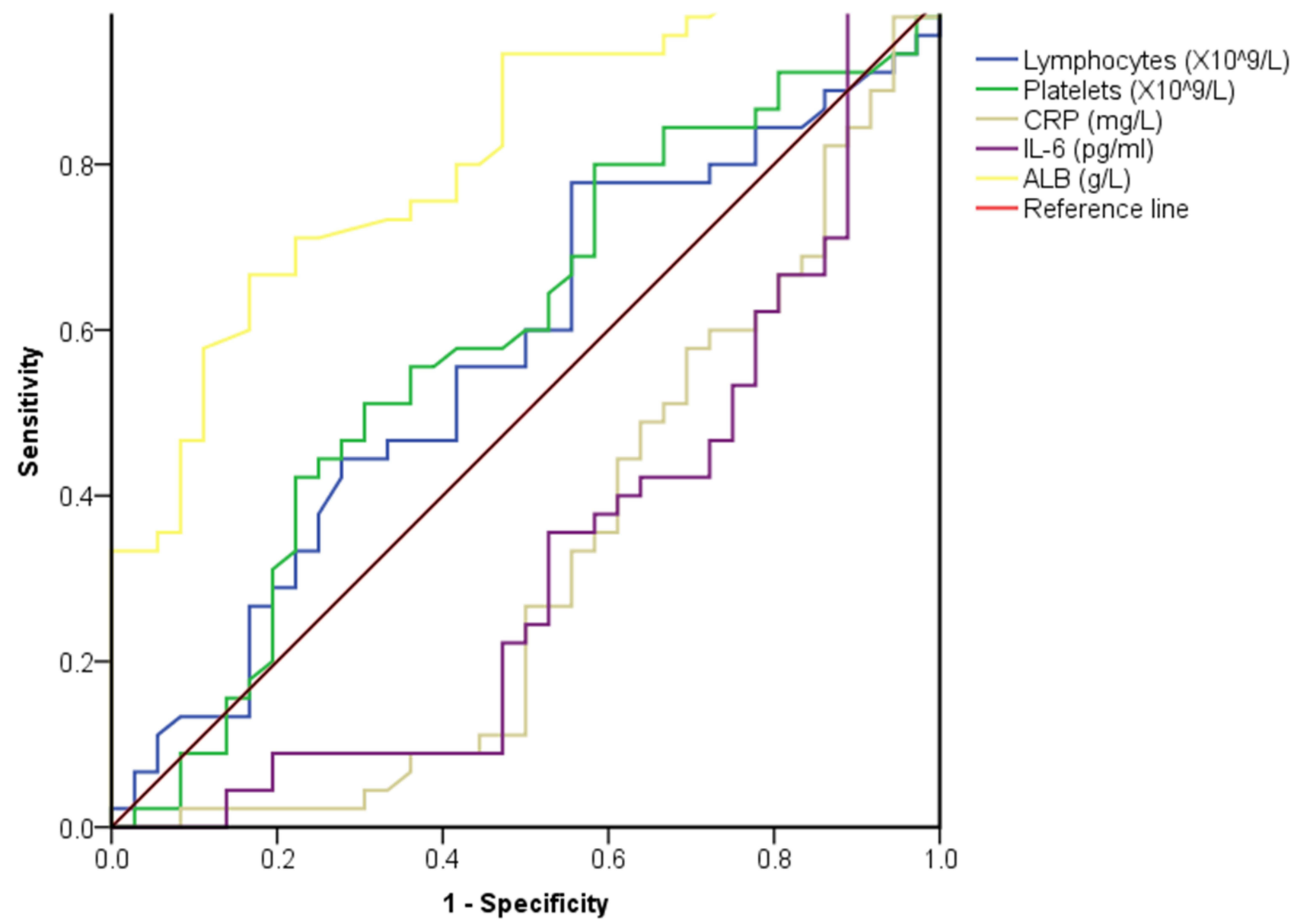

Figure 2 The Receiver Operator Characteristic (ROC) curves showing the performance of hematological parameters. The ROC curve analysis were performed on peripheral blood lymphocytes, platelets, CRP, IL-6 and ALB levels. The areas under the curve were calculated with the values of 0.57 ( $95 \%$ Cl: $0.44-0.70, p=0.294), 0.59$ (95\% Cl: 0.46-0.72, $p=0.174), 0.33$ (95\% Cl: $0.20-0.45, p=0.007), 0.33$ (95\% Cl: $0.21-0.45, p=0.009)$, as well as $0.8 \mathrm{I}(95 \% \mathrm{Cl}: 0.72-0.90, p<0.00 \mathrm{I})$, respectively. Abbreviations: ALB, Albumin; CRP, C-reactive protein; IL-6, Interleukin-6. 
$p=0.95)$. These results suggested that ALB may be a favorable prognostic factor for community populations with geographic differences, especially overseas Chinese and local Chinese patients.

\section{Discussion}

Multiple reasons have been demonstrated with the contributions to the geospatial discrepancy on COVID-19 spread $^{8-10}$ as well as the fatality. ${ }^{6,11}$ Here we showed that there existed dramatic differences in the inflammatory state and the clinical representations triggered by variable geographic distributions among local Chinese cases and overseas Chinese, as well as incoming foreign patients with SARS-CoV-2 infection. Mechanism studies have revealed that SARS-CoV-2 infection drives a unique and profound release of pro-inflammatory cytokines in the host, ${ }^{12,13}$ and thus the dysfunction of the intrinsic immune system may enhance the risk for severe SARSCoV-2 infection. ${ }^{14}$ Except for the ethnicity-specific characteristics in the host genetic level, it has been implied that the geographical differences in the epidemiological prevalence and clinical representations of COVID-19 patients may be derived from viral genetic variants. ${ }^{15}$ The recent work showed that the different strains of SARS-CoV-2 virus, mutated on spike protein with a shift from $\mathrm{D}$ to $\mathrm{G}$ at position 614 , possesses remarkable alterations in their current pathogenicity and transmissibility. ${ }^{16-18}$ In China, most samples are D614, while the viral samples of European and American cases are predominantly G614 variant, coordinately with a significant higher prevalence of chemosensory dysfunction than that of Asian patients. ${ }^{19}$ Some studies further demonstrated that the G614 strain was mainly associated with higher viral load and infectious titers, ${ }^{17}$ dispersing globally by travelers to different locations. ${ }^{20}$ Our current study revealed that incoming foreign patients and overseas Chinese patients exhibited lower levels of proinflammatory cytokines including IL-6 and CRP, juxtaposed to the attenuated viral elimination rate, as well as the prolonged hospital stay. Meanwhile local Chinese with confirmed COVID-19 experienced continuous improvements in virus removal capacity and a correspondingly shorter hospital stay. Therefore, our results confirmed the associations between diversity of clinical representations and geographic distribution of SARS-CoV-2 variants, and further indicated that the reduced viral clearance by G614 variant is probably associated with the attenuation of pro-inflammatory response in COVID-19 patients.

On the other hand, we also looked at the factors associated with geographic communities in the global COVID19 pandemic. Our results of ROC analysis showed that there was an association between serum ALB levels in COVID-19 patients and their geographic living environment. It suggested that a lower ALB level probably is an independent prognostic value for community populations from different geographic regions.

Above all, our study provides clues on distinguishable distinctions of inflammatory state and clinical representations between overseas Chinese and local Chinese in the progression of COVID-19 infection, which contributes to evidence-based practice to combat the global spread of SARS-CoV-2 infection. This work underscores the need to identify distinguishably prognostic factors of geographical dissimilarity in COVID-19 patients.

\section{Data Sharing Statement}

The data presented in this study are available on request from Dr. Pan, one of the corresponding authors. The data are not publicly available due to the protection of patient information.

\section{Acknowledgments}

This work was supported by Sanming Project of Medicine in Shenzhen (SZSM201512005).

\section{Author Contributions}

Shanglong Kou and Yanhua Liang contributed equally as co-first authors. Jing Yuan and Yanchao Pan are cocorresponding authors. All authors made substantial contributions to conception and design, acquisition of data, or analysis and interpretation of data; took part in drafting the article or revising it critically for important intellectual content; agreed to submit to the current journal; gave final approval of the version to be published; and agree to be accountable for all aspects of the work.

\section{Disclosure}

All authors declare that they have no conflict of interest exists.

\section{References}

1. Hoehl S, Rabenau H, Berger A, et al. Evidence of SARS-CoV-2 infection in returning travelers from Wuhan, China. $N$ Engl $J$ Med. 2020;382(13):1278-1280. doi:10.1056/NEJMc2001899 
2. Chinazzi M, Davis JT, Ajelli M, et al. The effect of travel restrictions on the spread of the 2019 novel coronavirus (COVID-19) outbreak. Science. 2020;368(6489):395-400. doi:10.1126/science.aba9757

3. Ceylan Z. Estimation of COVID-19 prevalence in Italy, Spain, and France. Sci Total Environ. 2020;729:138817. doi:10.1016/j. scitotenv.2020.138817

4. Grasselli G, Zangrillo A, Zanella A, et al. Baseline characteristics and outcomes of 1591 patients infected with SARS-CoV-2 admitted to ICUs of the Lombardy Region, Italy. JAMA. 2020;323 (16):1574-1581. doi:10.1001/jama.2020.5394

5. Zhou F, Yu T, Du R, et al. Clinical course and risk factors for mortality of adult inpatients with COVID-19 in Wuhan, China: a retrospective cohort study. Lancet. 2020;395(10229):1054-1062. doi:10.1016/S0140-6736(20)30566-3

6. Bialek S, Bowen V, Chow N; Team CC-R. Geographic differences in COVID-19 cases, deaths, and incidence - United States, February 12-April 7, 2020. MMWR Morb Mortal Wkly Rep. 2020;69 (15):465-471. doi:10.15585/mmwr.mm6915e4

7. Yuan J, Kou S, Liang Y, Zeng J, Pan Y, Liu L. Polymerase chain reaction assays reverted to positive in 25 discharged patients with COVID-19. Clin Infect Dis. 2020;71(16):2230-2232. doi:10.1093/ $\mathrm{cid} / \mathrm{ciaa} 398$

8. Gupta S, Raghuwanshi GS, Chanda A. Effect of weather on COVID-19 spread in the US: a prediction model for India in 2020. Sci Total Environ. 2020;728:138860. doi:10.1016/j.scitotenv.2020.138860

9. Jia JS, Lu X, Yuan Y, Xu G, Jia J, Christakis NA. Population flow drives spatio-temporal distribution of COVID-19 in China. Nature. 2020;582(7812):389-394. doi:10.1038/s41586-020-2284-y

10. Weiming T, Huipeng L, Gifty M, et al. The changing patterns of Coronavirus Disease 2019 (COVID-19) in China: a tempogeographic analysis of the severe acute respiratory syndrome coronavirus 2 epidemic. Clin Infect Dis. 2020;71(15):818-824. doi:10.1093/cid/ ciaa423

11. Pan A, Liu L, Wang C, et al. Association of public health interventions with the epidemiology of the COVID-19 outbreak in Wuhan, China. JAMA. 2020;323(19):1915-1923. doi:10.1001/jama.2020.6130
12. Blanco-Melo D, Nilsson-Payant BE, Liu WC, et al. Imbalanced host response to SARS-CoV-2 drives development of COVID-19. Cell. 2020;181(5):1036-1045. doi:10.1016/j.cell.2020.04.026

13. Azkur AK, Akdis M, Azkur D, et al. Immune response to SARS-CoV-2 and mechanisms of immunopathological changes in COVID-19. Allergy. 2020;75(7):1564-1581. doi:10.1111/all.14364

14. Schett G, Sticherling M, Neurath MF. COVID-19: risk for cytokine targeting in chronic inflammatory diseases? Nat Rev Immunol. 2020;20(5):271-272. doi:10.1038/s41577-020-0312-7

15. Butowt R, Bilinska K, Von Bartheld CS. Chemosensory dysfunction in COVID-19: integration of genetic and epidemiological data points to D614G spike protein variant as a contributing factor. ACS Chem Neurosci. 2020;11(20):3180-3184. doi:10.1021/ acschemneuro.0c00596

16. Li Q, Wu J, Nie J, et al. The impact of mutations in SARS-CoV-2 spike on viral infectivity and antigenicity. Cell. 2020;182(5):12841294 e1289. doi:10.1016/j.cell.2020.07.012

17. Volz E, Hill V, McCrone JT, et al. Evaluating the effects of SARS-CoV-2 spike mutation D614G on transmissibility and pathogenicity. Cell. 2021;184(1):64-75 e11.

18. Nguyen HT, Zhang S, Wang Q, et al. Spike glycoprotein and host cell determinants of SARS-CoV-2 entry and cytopathic effects. $J$ Virol. 2020;11:JVI.02304-20.

19. von Bartheld CS, Hagen MM, Butowt R. Prevalence of chemosensory dysfunction in COVID-19 patients: a systematic review and meta-analysis reveals significant ethnic differences. ACS Chem Neurosci. 2020;11 (19):2944-2961. doi:10.1021/acschemneuro.0c00460

20. Korber B, Fischer WM, Gnanakaran S, et al. Tracking changes in SARS-CoV-2 spike: evidence that D614G increases infectivity of the COVID-19 virus. Cell. 2020;182(4):812-827 e819. doi:10.1016/j. cell.2020.06.043
Infection and Drug Resistance

\section{Publish your work in this journal}

Infection and Drug Resistance is an international, peer-reviewed openaccess journal that focuses on the optimal treatment of infection (bacterial, fungal and viral) and the development and institution of preventive strategies to minimize the development and spread of resistance. The journal is specifically concerned with the epidemiology of
Dovepress

antibiotic resistance and the mechanisms of resistance development and diffusion in both hospitals and the community. The manuscript management system is completely online and includes a very quick and fair peerreview system, which is all easy to use. Visit http://www.dovepress.com/ testimonials.php to read real quotes from published authors 